\title{
Surface plasmon resonance optical sensor for detection of essential heavy metal ions with potential toxicity: copper, zinc and manganes.
}

\begin{abstract}
In this work, surface plasmon resonance (SPR) spectroscopy had been studied for detection of essential heavy metal ions with potential for toxicity: copper, zinc and manganese ions $(\mathrm{Cu} 2+, \mathrm{Zn} 2+$ and $\mathrm{Mn} 2+)$. The sensitivity of SPR optical sensor had been enhanced by introducing a thin crosslinked chitosan layer on a gold film. Crosslinked chitosan solution was synthesized by homogeneous reaction of medium molecular weight chitosan in aqueous acetic acid solution with glutaraldehyde as crosslinking agent. It was deposited on gold film by spin coating. From the results we found that the change in the resonance angle $(\Delta \theta)$ is directly proportional to the concentration of heavy metal ions solution $(0.5-100 \mathrm{ppm})$. The sensitivity of this optical sensor for the studied heavy metal ions are in following order: $\mathrm{Cu} 2+>\mathrm{Zn} 2+>\mathrm{Mn} 2+$. The gold/chitosan interface in SPR technique is highly sensitive for these heavy metal ions with detection limit as low as $0.5 \mathrm{ppm}$.
\end{abstract}

Keyword: Surface Plasmon Resonance; Crosslinked chitosan; Copper; Zinc; Manganese lons. 